\title{
Delayed Knee Locking after Complicated Anterior Cruciate Ligament Reconstruction Despite Good Stability Using A Bio-Transfix
}

\author{
Yong Seuk Lee, MD, PhD, Beom Koo Lee, MD, PhD, Jae Ang Sim, MD, PhD, Ji Hoon Kwak, MD and \\ Shin Woo Nam, MD \\ Department of Orthopaedic Surgery, Gachon University Gil Hospital, Gachon University School of Medicine, Incheon, Korea
}

\begin{abstract}
We describe a case of delayed cyst formation that presented as intermittent knee locking after complicated anterior cruciate ligament (ACL) reconstruction using a Bio-TransFix implant in a 21-year-old male patient. During femoral fixation, we could not pull out the guide wire that was temporarily used for the femoral fixation. However, stability was good, and the guide wire was not removed. This was shown to be a wrong type of fixation in a later study. During follow-up, the patient was satisfied and stability was relatively good until 18 months post-operatively. From the 2nd post-operative year, he experienced intermittent knee swelling and locking and pain around the lateral femoral condyle. Follow-up magnetic resonance imaging showed a large cyst around the broken wire tip, but the reconstructed ACL was fine.
\end{abstract}

Key words: Anterior cruciate ligament, Reconstruction, Complication, TransFix, Guidewire.

There are some options for femoral fixation of a hamstring or tibialis graft in an anterior cruciate ligament (ACL) reconstruction. Among them, the Bio-TransFix (Arthrex, Naples, FL, USA) implant is well-accepted and considered a strong option ${ }^{1)}$. However, this device is not easy to use and can cause some problems if caution is not exercised ${ }^{2,3}$. In our case, strong fixation was obtained using a Bio-TransFix implant intraoperatively, but the guide wire remained because we could not pull it out. This proved to be a wrong type of fixation in a later study $^{3)}$. The patient maintained stability, but suffered from a delayed complication that presented as intermittent knee locking

Received March 29, 2011; Revised June 20, 2011;

Accepted July 19, 2011.

Correspondence to: Beom Koo Lee, MD.

Department of Orthopaedic Surgery, Gil Medical Center, Gachon University, 1198 Guwol-dong, Namdong-gu, Incheon 405-760, Korea. Tel: +82-32-460-3384, Fax: +82-32-468-5437

Email: bklee@gilhospital.com

This is an Open Access article distributed under the terms of the Creative Commons Attribution Non-Commercial License (http://creativecommons.org/licenses/by-nc/3.0/) which permits unrestricted non-commercial use, distribution, and reproduction in any medium, provided the original work is properly cited.

Copyright ( ) 2011. KOREAN KNEE SOCIETY

www.jksrr.org originating from a delayed cyst formation. We report the cause and mechanism of this complication. This report provides information that may help others to prevent this complication.

\section{Case Report}

A 21-year-old man complained of painful instability in his right knee for several months after a twisting injury sustained while playing soccer. A physical examination and magnetic resonance image (MRI) showed a complete rupture of the ACL and a tear of the medial meniscus posterior horn (MMPH). An arthroscopic ACL reconstruction was performed using a hamstring autograft and an all inside repair of the MMPH was done. A BioTransFix implant was used for femoral fixation. During the femoral fixation, the graft was pulled to the femoral tunnel, and unrestricted back and forth motion of the wire (transverse motion that is an indirect sign of full proximal migration) was achieved. A dilator was inserted over the guide wire ${ }^{3)}$. The BioTransFix implant was inserted using the appropriate protocol and stability was checked. The stability was excellent but the guide wire could not be pulled out and was found to be broken at the end. This is because the bio-TransFix implant and medial side wire co-existed in the predrilled narrow space, which is located in the medial side of the femoral tunnel. If the wire is removed, the 

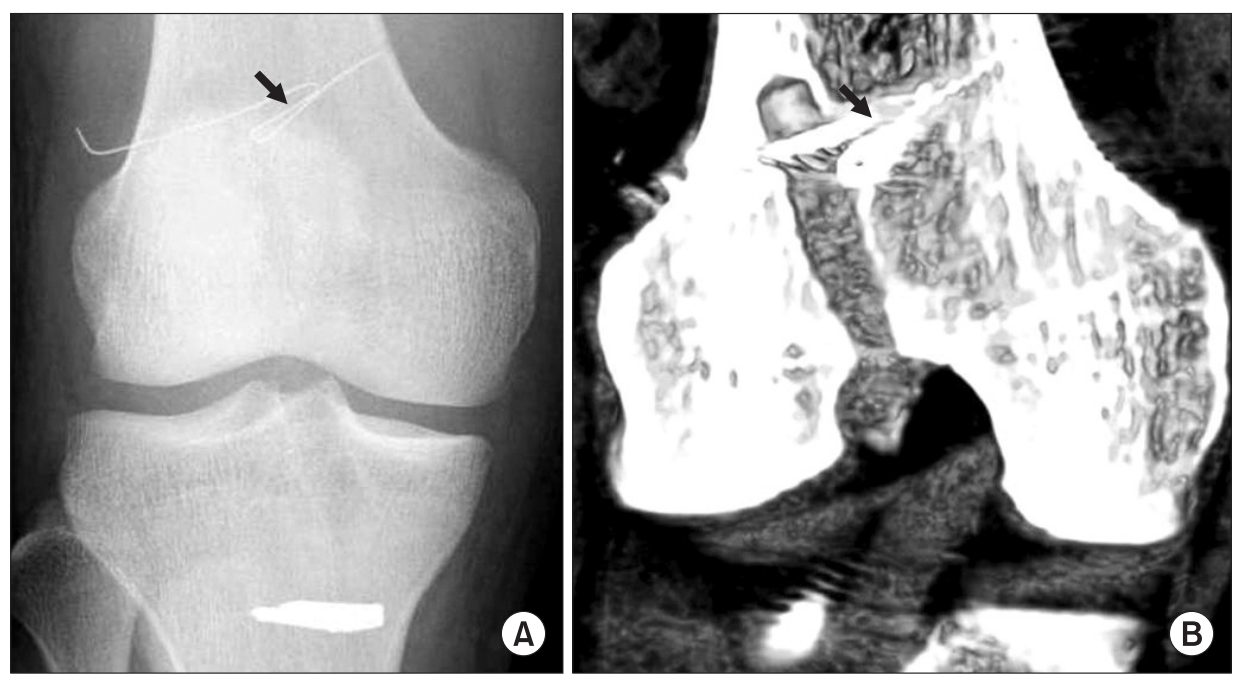

B
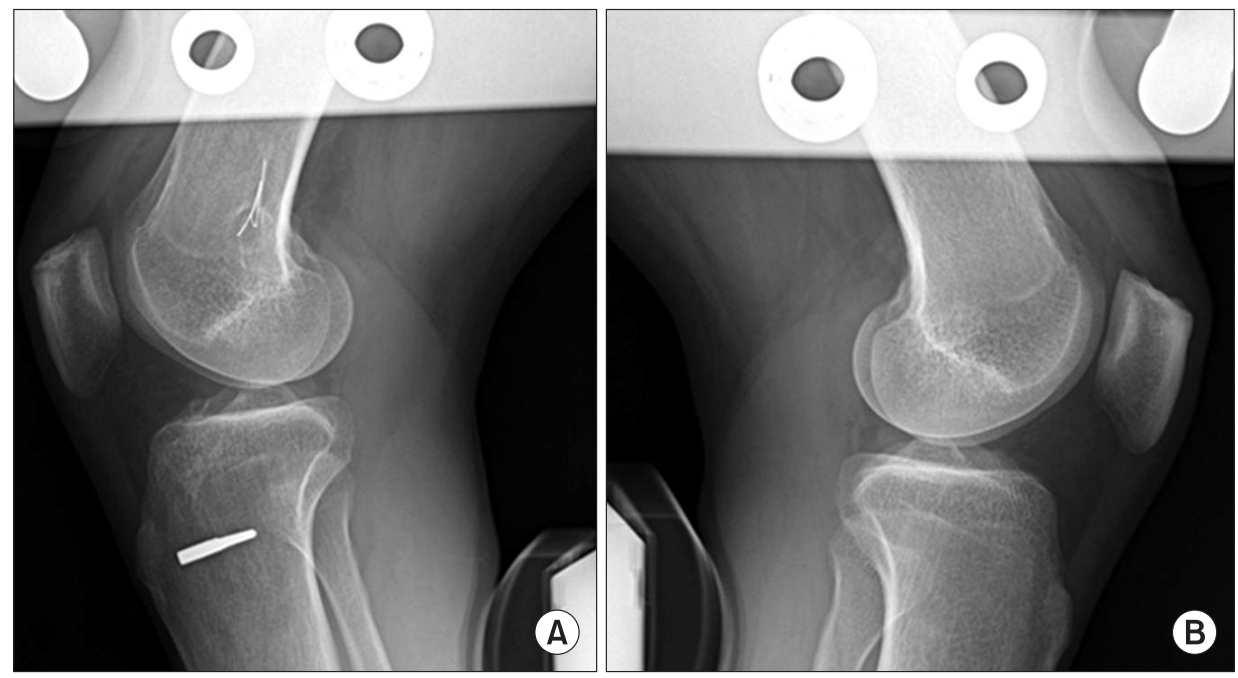

Fig. 2. Stress radiographs show a $1 \mathrm{~mm}$ difference between the reconstructed (A) and the normal knee (B).
Fig. 1. Postoperative plain anterior posterior radiograph shows the remaining broken wire on the femoral side (A). The CT scan using the ligament setting to remove the metal artifact shows no graft on the Transfix implant but fixation only to the wire loop (B). Arrow: broken guide wire.
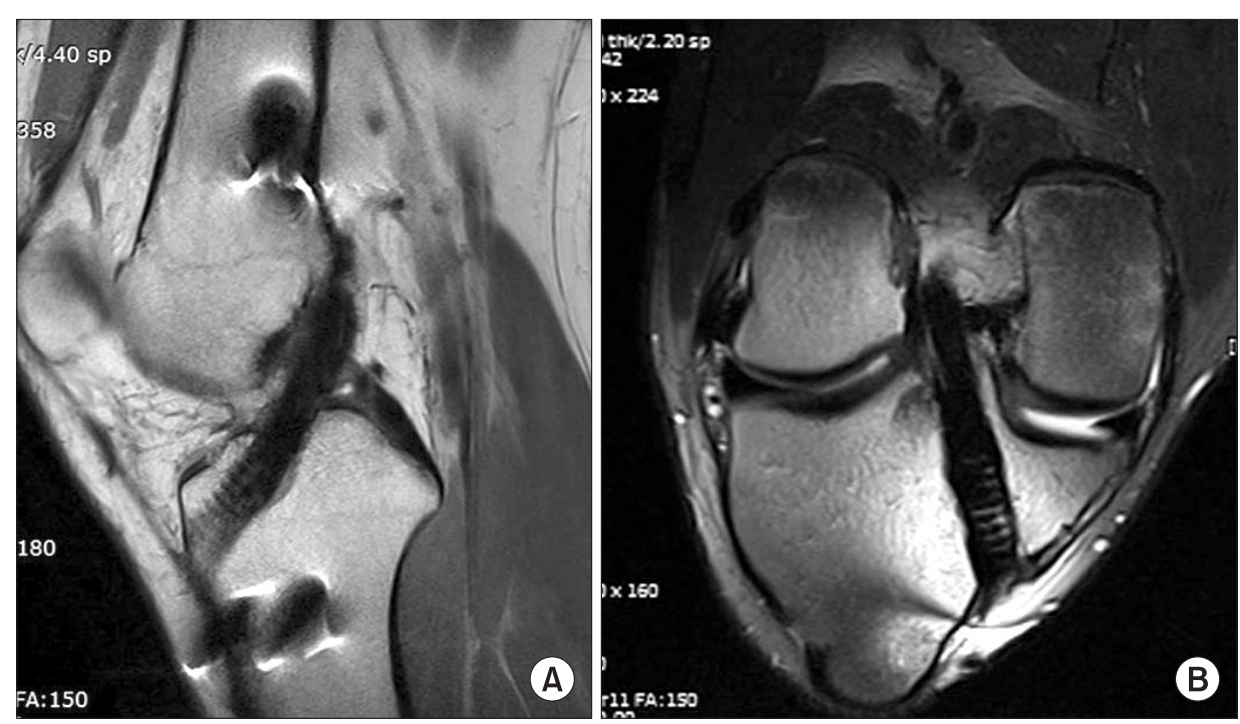

Fig. 3. MR images (A: sagittal, B: oblique coronal) show a dark and straight graft signal of the reconstructed anterior cruciate ligament. 

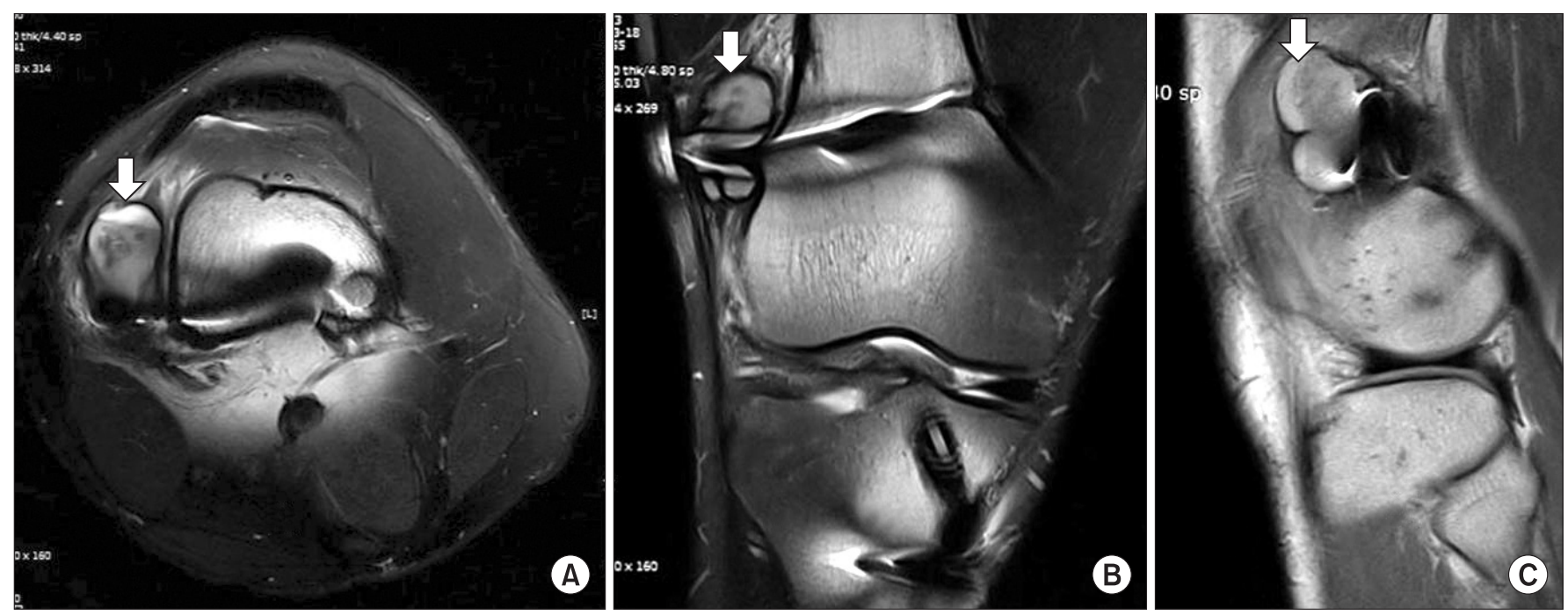

Fig. 4. MR images (A: axial, B: coronal, and C: sagittal) show a $3 \times 3 \mathrm{~cm}$ cystic lesion at the lateral aspect of the right distal femur, just adjacent to the metal wire tip. Arrow: cyst around broken guide tip.

graft can not be fixed with any approach. The TransFix implant must be inserted between two strands. However, the implant in our case was inserted above the loop portion of the graft. A broken wire that exits medially is compressed by the forehead portion of the Bio-TransFix implant and the graft is hung on the wire loop because the Bio-TransFix implant and medial side two wire co-exist in the predrilled narrow space located on the medial side of the femoral tunnel (Fig. 1$)^{3}$. During the operation, a 1 $\mathrm{cm}$ incision was used for the TransFix insertion. We maintained the guide wire because fixation failure could occur if the wire was pulled. Therefore, we cut the wire via a skin incision for the TransFix insertion as maximally as possible. The patient underwent routine post-operative rehabilitation and the early postoperative course was not eventful.

During follow-up, the patient was satisfied and stability was luckily good until 18 months post-operatively. From the 2nd post-operative year, he experienced intermittent knee swelling, locking and pain around the lateral femoral condyle. Stability was also excellent at this time (Fig. 2) and a well-reconstructed ACL was visible (Fig. 3). However, an abnormal $3 \times 3 \mathrm{~cm}$ cyst around the broken wire tip was observed in MRI (Fig. 4), which originated from the tip of the broken wire. It was thought that some irritation had occurred from the tip of the TransFix implant, and this was the cause of the locking symptom. The cyst was excised and the wire and TransFix were also removed. Tendon to bone healing was achieved because stability was also good after removal.

\section{Discussion}

In the analysis of complications of ACL reconstruction using a Transfix implant, the mechanism of such good stability with incorrect fixation was considered to be broken wire loop fixation. The cause of this complication was examined, the surgical procedure was checked, and a tight femoral tunnel was suspected. The possibility of an incomplete full proximal insertion of the graft was suspected. The guide wire is relatively flexible compared with the Bio-TransFix implant. Therefore, unrestricted back and forth motion of the wire can occur even though the wire is mildly curved $^{3}$. Hence, unrestricted back and forth motion of the wire does not confirm the full proximal insertion of the graft.

Complications of the use of Transfix implants have been documented previously ${ }^{4-6)}$. Pelfort et al. ${ }^{6}$ reported the presence of iliotibial band friction syndrome in two cases using the BioTransfix femoral fixation device. The implants extruded laterally and the implant-tail was broken in both cases. Kokkinakis et al. ${ }^{5}$ also reported a case of wire breakage that also showed good stability. As reported by Lee et al. ${ }^{7}$, there is some possibility of a delayed foreign body reaction to the Bio-Transfix implant. However, in our case, the cyst may have been due to the irritation caused by the broken metal tip because the cyst formed just adjacent to the metal wire tip and the Transfix implant was intact and well-fixed at the original site (Figs. 1, 4). This means that this complication can be avoided if an operation is performed with the right understanding of the mechanism of the implant and proper surgical technique.

For prevention of this complication, we analyzed complications 
and proposed some solutions. First, vertical traction is helpful for full proximal insertion of a graft regardless of the unrestricted back and forth motion. Second, the thigh portal that is made at the exit portion of the beath pin for the femoral tunnel should be also used. With this portal, this complication can be avoided and controlled by observing the fixation. The view from this portal allows femoral fixation directly and biting of the implant or dilating instrument can be observed at the point of insertion ${ }^{8)}$.

From this case we learned that good stability obtained through the use of an abnormal method (wrong fixation) can cause unpredicted complications. Therefore, we should understand the correct mechanism of using the Bio-TransFix implant.

\section{References}

1. Misra R, Strover A, El-Shazly M. Intra-articular protrusion of malpositioned Transfix implant following anterior cruciate ligament reconstruction. Arthroscopy. 2006;22:226. e1-e4.

2. Choi NH, Son KM, Victoroff BN. A pitfall of transfix fixation during anterior cruciate ligament reconstruction. Knee Surg Sports Traumatol Arthrosc. 2008;16:479-81.

3. Jung YB, Lee YS, Jung HJ, Nam CH, Yang JJ. Correction of bony genu recurvatum combined with ligamentous instability of the knee: three case reports. Knee Surg Sports
Traumatol Arthrosc. 2008;16:185-7.

4. Jaberi FM, Haghighat A, Babanezhad Z, Jaberi MM. Arthroscopic anterior cruciate ligament reconstruction with modified "wireless" cross-screw transfix femoral fixation. Knee Surg Sports Traumatol Arthrosc. 2010;18:1508-10.

5. Kokkinakis $\mathrm{M}$, Ashmore A, El-Guindi M. Intraoperative complications using the Bio-Transfix femoral fixation implant in anterior cruciate ligament reconstruction. Arch Orthop Trauma Surg. 2010;130:375-9.

6. Pelfort X, Monllau JC, Puig L, Caceres E. Iliotibial band friction syndrome after anterior cruciate ligament reconstruction using the transfix device: report of two cases and review of the literature. Knee Surg Sports Traumatol Arthrosc. 2006;14:586-9.

7. Lee BI, Yoo JH, Chun DI, Choi HS, Min KD, Jeen YM. Delayed foreign body reaction due to bioabsorbable pins used for femoral fixation in anterior cruciate ligament reconstruction: a case report. Am J Sports Med. 2010;38: 176-80.

8. Lee YS, Ahn JH, Kim JG, Park JH, Park JW, Kim CB, Lee SW. Analysis and prevention of intra-operative complications of TransFix fixation in anterior cruciate ligament reconstruction. Knee Surg Sports Traumatol Arthrosc. 2008;16:639-44. 